H I G H L I G H T S

MICROBIAL GENOMICS

\section{Breaking down S. coelicolor}

If you took antibiotics this winter to shift a troublesome sore throat, it's likely that they were produced by a relative of Streptomyces coelicolor - a soil-dwelling bacterium with an unusual talent for breaking down complex substances. Now, a team of UK and Taiwanese researchers report in Nature the sequencing of the genome of S. coelicolor A3(2), providing a first glimpse of the wealth of genes that underlie this bacterium's complex lifestyle and environmental adaptability. Importantly, this team have discovered previously unreported metabolic enzymes that could be put to good use to engineer new and much-needed antimicrobials.

The first surprise to emerge from S. coelicolor's genome is the number of genes present on its linear chromosome - 7,825 - nearly double the number found in the related bacterium Mycobacterium tuberculosis. This large number of genes is predominantly due to the presence of large protein families, as well as many new ones, which appear to be mostly involved in the regulation, transport and degradation of extracellular nutrients. And the surprises don't stop there, as S. coelicolor also has an unprecedented number of regulatory genes - mostly required to respond to stress and environmental stimuli — and 20 chromosomal gene clusters that encode enzymes predicted to function in secondary metabolism.

Although genes are distributed evenly over the S. coelicolor chromo-

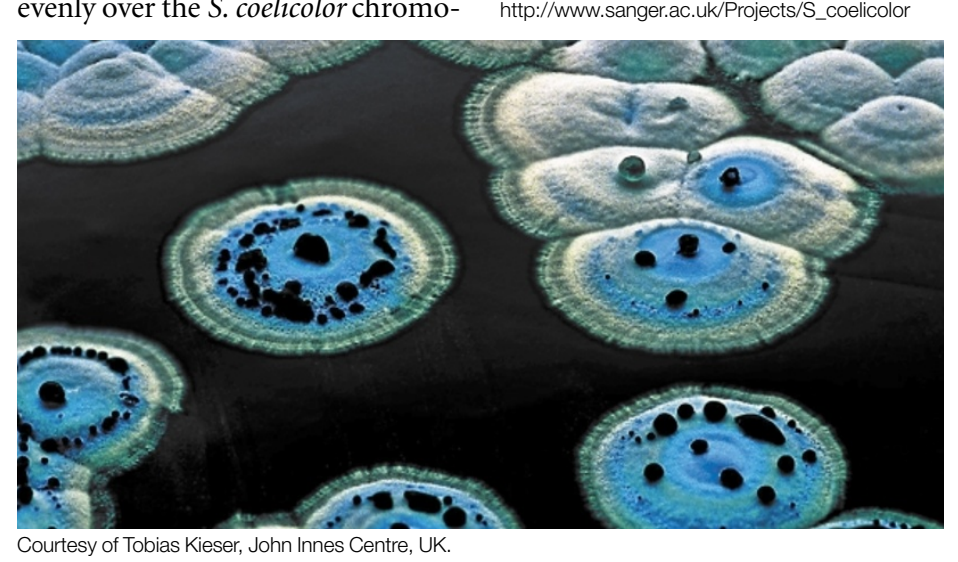

some, a biphasic structure emerges when they are categorized according to their predicted functions - essential genes (such as those involved in cell division, transcription and translation) cluster in a central core, whereas 'contingency' genes, those with non-essential functions, are located on the chromosome's arms. The reason for this structure became clear when the authors compared S. coelicolor's genome with that of two other actinomycetes, M. tuberculosis and Corynebacterium diphtheriae. The core of S. coelicolor's genome is highly conserved with the genomes of these two pathogens, indicating their evolution from a common ancestor. However, the arms of S. coelicolor are not conserved and appear to be remarkably tolerant to genome rearrangements and insertions - the authors found evidence that 14 regions have recently been introduced to the genome by lateral transfer, adding to the bacterium's repertoire of metabolic enzymes.

Given this bacterium's medical importance - its close relatives produce two-thirds of all naturally derived antibiotics and many other pharmaceuticals, including antitumour agents and immunosuppressants - its genome is a tremendously important resource. Once the biological secrets of the newly discovered metabolic enzymes have been broken down, they can be used to engineer metabolic pathways to produce new therapeutic compounds.

\section{(4) References and links} ORIGINAL RESEARCH PAPER Bentley, S. D. et al. Complete genome sequence of the mode actinomycete Streptomyces coelicolor A3(2). Nature 417, 141-147 (2002) WEB SITE

S. coelicolor genome project: http://www.sanger.ac.uk/Projects/S_coelicolor

\section{IN BRIEF}

\section{EPIGENETICS}

A genome-wide screen for normally methylated human CpG islands that can identify novel imprinted genes.

Strichman-Almashanu, L. Z. et al. Genome Res. 12, 543-554 (2002)

Imprinted genes are often associated with methylated $\mathrm{CpG}$ islands, unlike the rest of the genome, where $\mathrm{CpG}$ dinucleotides are thought to be largely unmethylated. The authors have used a restriction-enzyme-based cloning strategy to screen systematically the human genome for unique, methylated $\mathrm{CpG}$ islands. At least two of the mapped clones are close to imprinted genes, validating this approach as a way to find new imprinted genes in mammals.

\section{DEVELOPMENTAL BIOLOGY}

Modulation of CREB activity by the Rho GTPase regulates cell and organism size during mouse embryonic development.

\section{Sordella, R. et al. Dev. Cell 2, 553-565 (2002)}

Rho GTPases regulate embryonic tissue morphogenesis and various key cellular signalling pathways. A knockout of a putative Rho inhibitor, p190-B RhoGAP, phenocopies mice without CREB - they are 30\% smaller than normal and are developmentally defective. Mutant cells have abnormally high levels of active Rho, are small and fail to activate CREB in response to insulin or IGF1, indicating that these GTPases modulate an insulin/IGF1 signal to CREB that determines cell and animal size.

\section{PLANT GENETICS}

A MADS-box gene necessary for fruit ripening at the tomato Ripening-inhibitor (Rin) locus.

Vrebalov, J. et al. Science 296, 343-346 (2002)

Fruit species can be divided into those, such as tomatoes, that require the gaseous hormone ethylene to ripen, and those that do not. Tomato rin (ripening-inhibitor) mutants do not ripen due to defects upstream of the ethylene/non-ethylene-mediated ripening control. This paper reports that the rin phenotype results from the fusion of tandem transcripts that encode MADS-box transcription factors: LeMADS-RIN, which controls ripening, and LeMADS-MC, which controls sepal development.

\section{GENE EXPRESSION}

Precision and functional specificity in mRNA decay.

Wang, Y. et al. Proc. Natt. Acad. Sci. USA 99, 5860-5865 (2002)

Wang et al. have used microarrays to measure the decay of mRNAs transcribed from over 4,000 genes in Saccharomyces cerevisiae after shutting off transcription by inactivating a temperature-sensitive RNA polymerase II. They find that decay rates vary widely and do not correlate with RNA abundance or ORF size, among other factors. However, mRNAs that encode proteins that function in stoichiometric complexes have similar turnover rates. mRNA turnover therefore might be linked to the physiological function of the encoded protein. 